$\mathrm{DSF}-29 / 2006$

\title{
HOLE THEORY AND QUANTUM ELECTRODYNAMICS IN AN UNKNOWN FRENCH MANUSCRIPT BY ETTORE MAJORANA
}

\author{
S. ESPOSITO
}

\begin{abstract}
We give an accurate historical and scientific account of a previously unknown manuscript written by Ettore Majorana in French. The retrieved text deals with Quantum Electrodynamics by using the formalism of field quantization, and it is here reported, for the first time, in translation. It is likely related to an invited talk for a conference at Leningrad (or Kharkov) in 1933 (or 1934) which, however, Majorana never attended. Probably this manuscript refers to the last missing papers of the "Senatore folder", given by Majorana to one student of his at the University of Naples in 1938, just before his disappearance.
\end{abstract}

\section{The LAST PAPERS WRITTEN By MAJORANA}

Ettore Majorana, born on 5 August 1906, taught from January to March 1938 at the University of Naples, having obtained the full professorship in Theoretical Physics "for high and well-deserved fame" 1, 2. The interest in the course held by the great Italian physicist, the only one effectively delivered to students [3], has been already pointed out in the literature (see, for example, Refs. 4, [5]). It is fundamentally based on the forefront feature with which Majorana characterized his teaching of Quantum Mechanics. The historical and personal adventure of Majorana, instead, is centered around his mysterious disappearance at the end of March 1938, exactly during his stay in Naples. On Friday 25 March (Majorana held his 21st lesson the day before), according to one of his student, «contrary to what he usually did, Majorana came to the institute and remained there for few minutes... From the corridor leading to the small room where I was writing, he called me by name: 'Miss Senatore...'. He didn't enter in the room but remained in the corridor; I went to him, and he gave me a closed folder, telling me: 'that's some papers, some notes, take them.... We will talk about them later'. Afterwards he went away and, turning around, said again, 'we will talk about them later'.» 6. That folder contained the lecture notes for the course on Theoretical Physics prepared by Majorana who, in that occasion, wanted to give once and for all to one of his student. What remains of the original papers with those notes was published some years ago 77. However, when Senatore looked at such publication, after very many years since 1938, she pointed out that «some chapters of the lectures are missing; their text (completed by the notes of the lecture the professor would have delivered on the day after his disappearance) was given to me. A few other sheets are still missing; these were written in the same original and neat way as the others, but they do not correspond to the lectures he had already given $\gg$. The path followed by the original notes by Majorana from 1938 to 1966, when Edoardo Amaldi deposited them at the Domus Galilaeana in Pisa together with the other scientific (unpublished) papers 
by Majorana, has been quite tortuous, as pointed out elsewhere [4. Thus it could be not surprising that some "chapters" have been "lost". Nowadays, however, we may safely assert that the whole set of the lecture notes for the Theoretical Physics course have been recovered [8], thanks to the recent discovery of the Moreno Paper 44. This document contains the notes corresponding to six lectures of the given course, whose original manuscripts are missing.

Instead, with regard to the notes corresponding to what Senatore interpreted as "the lecture the professor would have delivered on the day after his disappearance", a very recent analysis [9] has concluded that they do not refer at all to a lecture of that course of Theoretical Physics, but rather to the text of a general conference or seminar probably addressed to the researchers of the Institute of Physics in Naples itself, certainly not devoted to students.

\section{The Senatore Folder}

About the remaining "few other sheets", strangely up to now no fruitful research has been conducted, although a long period elapsed since the time when the Majorana papers were made publicly available. Such a research is performed now, based mainly on the papers at the Domus Galilaeana, and the results are here presented for the first time.

The folder with the Naples lecture notes given to Senatore on 25 March 1938 contains now the following documents.

Firstly, the original of a letter written by Gilberto Bernardini to Amaldi on 2 December 1064 (reproduced in Ref. [7) is present in it. In this letter Bernardini replied to Amaldi about his persistent request on the Majorana lecture notes. Evidently, such a letter was added by Amaldi in recent times (around 1966) to the folder.

The second document ${ }^{1}$ present in the folder is composed by $22 \mathrm{~A} 4$ papers (each of one corresponding to 4 pages). They are the original notes corresponding to 10 lectures of the Majorana course [8].

The third document is, instead, composed of only 3 papers (each of one corresponding again to 4 pages). The text contained in it refers to what mentioned above regarding the general conference delivered by Majorana (see Ref. 9]). For possible future reference, this document of 3 papers will be denoted as "excerpta Senatore I".

Unexpectedly, the folder at the Domus Galilaeana contains also another document, composed of 2 A4 papers (the first one made of 4 pages, while the other one of only two pages). Such a document, denoted here as "excerpta Senatore II", is completely different from the excerpta Senatore I (and from the lecture notes), due to peculiarity of the text, which is written in French rather than in Italian, and to particular writing details.

The content of the text of this manuscript, reported (translated into English) in the appendix, concerns about an application of the formalism of second quantization to the Dirac's hole theory (excerpta Senatore IIa) and some topics of Quantum Electrodynamics (excerpta Senatore IIb e IIc), which will be discussed from a theoretical point of view in the following section. However, it is interesting to observe

\footnotetext{
${ }^{1}$ The subdivision in terms of "documents" is made here for convenience, but it does not correspond to any real division. Indeed, the folder contains only spare sheets, according to what here described.
} 
at once that, despite the apparent variety of topics, the whole French text present in the 2 papers seems to have been written in no more than one occasion.

\section{The RefERence theoretical BaCkground}

In the general framework of Wave Mechanics, basically introduced by Erwin Schrödinger, the existence of a "matter quantum", such as the electron, posed the same theoretical problems of those implied by the existence of light quanta in the context of the Maxwell electromagnetic theory. This was clearly noted by Werner Heisenberg in 1929 [10. Indeed, if the fundamental problem related to the existence of the photons was that of explaining the interference phenomenon, for the electrons it was that of deducing the quantization of the electric charge starting from the Schrödinger wavefunction.

As well known, the solution to such difficulties came out with the introduction of the field quantization (or "second quantization") procedure that, after a preliminary work by Pascual Jordan in 1926 [1], it was applied for the first time by Paul A. M. Dirac in 1927 [12] to the quantization of the electromagnetic field. The following step was that of considering particles with non vanishing mass, and this was achieved by Jordan and Oskar Klein [13], who generalized the Dirac quantization method to bosonic fields. The procedure to be adopted for fermions, according to the Pauli exclusion principle, required instead the "invention" of anticommutators, as pointed out by Jordan and Eugene Wigner in 1928 [14].

The key point was, evidently, the realization that the (Schrödinger, Dirac or Maxwell) wave field does not represent a single particle but, on the contrary, an arbitrary number of particles. To this regard, it is particularly expressive the title given by Jordan and Klein to their fundamental paper, On the many-body problem of the Quantum Theory.

The inclusion of the interaction between light and matter in the second quantization procedure was systematically considered in 1929-1930 by Heisenberg and Wolfgang Pauli [15], who elaborated a first theory of Quantum Electrodynamics by using the lagrangian formalism. In this theory, however, some fundamental problems began to impose to the attention of the theorists, such as the emergence of an infinite Coulomb self-energy for the electron and the problem of the negative-energy states (that is the transition between states with energy $E=+m c^{2}$ to states with $\left.E=-m c^{2}\right)$. Despite the engagement of some of the best theoretical physicists of that epoch, such problems remained unsolved for a given period of time. In particular the problem of the negative energy states increased when Ivar Waller [16] proved that they were effectively required in order to obtain the Thomson classical limit of the scattering cross section of X-rays from atoms. The Dirac theory of "holes" [17, interpreted earlier in terms of protons (by following an Hermann Weyl's conjecture [18) and then assuming the existence of positrons as subsequently observed, offered a simple and interesting solution to the problem, as well known. It was thus introduced the concept of "vacuum" (or, using the words by Dirac, "the normal state of electrification") as a sea of electrons where all the positive energy states are empty, whilst those with negative energy are all occupied.

The general acceptance of the hole theory was, initially, not unquestioned inside the physicists' community, and still in 1933, after the experimental discovery of the "antielectron", Pauli expressed in many occasions his perplexity about it 19. A completely different viewpoint was instead adopted by Heisenberg who 
was favorably impressed by the substantial symmetry, in the Dirac theory, between processes involving electrons and those involving positrons [20], an idea that will be later formally recovered by Majorana in his famous article on the Symmetrical theory of electrons and positrons 21] (whose genesis could, probably, be dated to 1933, when Majorana visited Heisenberg in Leipzig). Heisenberg himself, in fact, in 1931 elaborated a particularly interesting (for our aims) application, in which he considered the symmetry between holes and electrons in an occupied atomic level or in an occupied energy band of a crystal [22] (that is: an application to an actual case, in contrast to the "vacuum" by Dirac). In this paper Heisenberg obtained a second quantized hamiltonian expressed in terms of holes (see Eq. (15) in Ref. [22]), where some additional one-particle operator terms comes out from the commutation between two-particle operators. The application to the case of an atomic level of $N$ electrons containing, however, only $n$ electrons was studied in the first part of the article, and led to a wave equation (Eq. (17) in Ref. 22]) for $N-n$ holes. In the second part of it, the electron-hole symmetry in the case of a metal with an "anomalous" Hall effect is instead considered. As already mentioned, Heisenberg used the formalism of second quantization, by making explicit reference to both the general article by Jordan and Klein of 1927 [13] and to that of Jordan and Wigner of 1928 14, where the anticommutators for fermions (namely, electrons and holes) were properly introduced.

In the excerpta Senatore IIa Majorana substantially followed the track left by Heisenberg in 22, but it is not clear if the author referred to an actual case (as that of Heisenberg) or, even more interestingly, if his intention was that of formulating a general theory of holes. Indeed, in the opening of the manuscript, the general assumptions to which Majorana appealed were very clearly and explicitly asserted, together with the reference point of the method of the "quantization of the Schrödinger equation" (i.e. that of second quantization). As well as Heisenberg, Majorana obtained the expression of the hamiltonian of the system in terms of holes, but it is evident that the theory elaborated in this manuscript is incomplete (or, rather, interrupted)

It is also particularly interesting the "justification" that Majorana gave for the adoption of anticommutators instead of commutators, which he referred to the particular form of the hamiltonian and to the corresponding equations of motion to be satisfied.

In the excerpta Senatore IIb and IIc, ${ }^{2}$ instead, Majorana considered a topic that he frequently studied in his personal research notebooks [23]: if, as recalled above, the Maxwell theory of electromagnetism should be viewed as the Wave Mechanics of the photon, then it should be possible to write the Maxwell equations as a Diraclike equation for a suitable wavefunction. An alternative model to the theory of Electrodynamics, based on an analogy with the Dirac theory of the electron, was already proposed by Oppenheimer in 1931 24; the contribution given by Majorana to this subject has been already discussed elsewhere 25], and we refer there the interested reader for further inputs related to the second part of the manuscript considered.

Regarding the excerpta Senatore IIc it is instead particularly interesting to point out the clear and explicit presentation of the experimental properties of photons,

\footnotetext{
${ }^{2}$ Although the same topic is treated in these two pages, it is preferable to introduce a further subdivision into two parts, since it is evident the lack of connection between them.
} 
upon which the novel formulation of Electrodynamics may be based, related to the velocity, energy, momentum and spin of photons. Note also that the Dirac-like equation for the photons reported at the end of the IIc manuscript is substantially different from that considered in the IIb one (and by Oppenheimer). It is applied to a two-component wavefunction (corresponding to the only two different polarization states of the photon) instead of a three-component one (corresponding to the electric and magnetic fields of the associated wave).

\section{The composition of the manuscript and a Soviet conference}

Unfortunately, the excerpta Senatore are not dated, so that it is difficult, without suitable assumptions, an accurate analysis of the genesis and composition of the Majorana work here considered. However, some "reasonable" considerations may be usefully developed, without forgetting the fundamental point just pointed out.

First of all, based on the kind of writing used, we can certainly exclude a composition date around that for the university lectures at Naples (1938). Indeed, from a comparison with other documents present in the archive of the Domus Galilaeana, especially the original manuscripts of the published articles by Majorana, we deduce that some writing details are very similar to those found for the paper in Ref. 26] of $1932 .^{3}$

The analysis of the specific contents of the Majorana manuscript seems to confirm a composition date not prior than 1932 revealing, for the excerpta Senatore IIa, a certain dependence on the Heisenberg article in [22] and, for the excerpta Senatore IIb e IIc, at least the knowledge of the Oppenheimer paper of the same year 24].

These only data, however, lead to generic conclusions, pointing out only a lower limit on the composition date, and certainly they do not offer hints on the genesis and the expected use of the manuscript.

Looking at the whole set of papers left by the Italian physicist, conserved not only in public archives like the Domus Galilaeana, but also in the private one of the Majorana family, we have found that only another document was written in French. It corresponds to a draft of a letter by Majorana in replying to an invitation to a conference (see the document MX/R1 in 11). In the following we give the translation of it:

Dear Sir,

I thank you warmly for your invitation to participate to the next congress that will take place in Leningrad. I am happy to accept and to have the occasion of visiting, at the same time, your big and beautiful country. I have talked about your invitation with Mr. Fermi and Mr. Rossi. Fermi is engaged in a course of conferences in America and cannot participate. Rossi, on the contrary, assured me that will accept with pleasure to go to Russia.

Dear Sir, receive my hearty greetings together with my warmest thanks.

Yours

Ettore Majorana

\footnotetext{
${ }^{3}$ They are also similar to those of the article in Ref. [27] of 1928, which would denote a composition preceding the Heisenberg paper of 1931 [22]. Although such an hypothesis cannot be completely excluded, based on what noted in the previous section and to what discussed below, we consider it not very likely.
} 


\author{
Ettore Majorana \\ Institute of Physics of the R. University \\ Rome \\ Bruno Rossi \\ Institute of Physics of the R. University \\ Padua
}

Although even this document is not dated, we can deduce some interesting information from what reported in it, if properly examined.

First of all, the letter containing the invitation to the conference was directed to Majorana as well as to Bruno Rossi (and Enrico Fermi), who worked at that time at the Institute of Physics in Padua. Now, it is known that Rossi was in Padua since the Fall of 1932, and that in between 1932 and 1933 he worked together with Fermi (in Rome) on some questions about Cosmic Rays Physics (see the paper in 28] and its presentation in the book [29] at page 509).

The second important fact regards the mentioned conference, apparently to be held in Leningrad. In the years considered, the only one conference of interest for us is the First All-Union Conference on Nuclear Physics [30, whose main topic was the Physics of atomic nucleus. It was held on 24-30 September of 1933 at the Leningrad Physico-Technical Institute 31. Contrary to what asserted in the letter above, neither Majorana nor Rossi participated to such a conference (as well as to other ones in the Soviet Union) while, among the others (for example, I.E. Tamm, V.A. Fock, G.A. Gamov, P.A.M. Dirac, F. Joliot, V.F. Weisskopf) Franco Rasetti, another member of the "Via Panisperna boys" group, took part to it 31. It is even interesting to observe that Dirac participated to this conference with a talk on the "Theory of the positron" 32, that is exactly the hole theory considered by Majorana in the mentioned paper.

The date of the Leningrad conference is in agreement also with another important piece of information contained in the Majorana letter, regarding the absence of Fermi who was "engaged in a course of conferences in America". Indeed, in the August 1933 Fermi, accompanied by Emilio Segrè, went to the summer school at Ann Arbor 33] 34, where he was invited to deliver some lectures (the same happened in 1930 and 1935).

Nevertheless the hypothesis just considered is not free from some difficulties, concerning mainly who effectively suggested to invite Majorana and Rossi to the conference and when the replying letter was effectively written by Majorana, after that the invitation arrived in Rome. In fact, regarding the first point, the work by Majorana (and, partially, that of Rossi) was not yet sufficiently known by the international community at the beginning of 1933. Instead, for the second point, we have to recall that Majorana was in Leipzig (and in Copenhagen, but not in Rome) from January to the beginning of August of 1933 [1], except for the Easter holidays ranging from the end of April to the beginning of May of that year. Then it seems unlikely that a visit to Leningrad could be organized only one month before the conference (in August) or in the short period of the Easter holidays.

The international fame of Majorana in the field of Nuclear Physics and, more in general, in that of Theoretical Physics, increased only after his stay in Leipzig. Incidentally, we note that Heisenberg publicized a lot the Majorana work (especially 
that on the nuclear exchange forces [35]) in the conferences following the mentioned visit [36] and, in particular, in the Solvay Congress at Bruxelles of the October 1933, where talks were given in French. The invitation to Majorana and Rossi (Heisenberg himself, who worked in the field of Cosmic Rays Physics too, was as well a quite good admirer of the Rossi's papers on this) could then be conceived in this occasion, and then formalized later.

Taking for grant such an hypothetical occurrence, the conference cited in the Majorana letter cannot be that of September 1933 in Leningrad. The subsequent conference in soviet soil was not given in this town, but in the other important center of Kharkov in May 1934 [37] 38. It was, nevertheless, organized by important Russian physicists working in Leningrad, such as A.F. Joffe and others. Few nonRussian physicists took part to this International Conference of Theoretical Physics (then, a conference not explicitly limited to Nuclear Physics); among the others, we mention Niels Bohr, Leon Rosenfeld (both were known to Majorana since its 1933 visit to Copenhagen), Ivar Waller and Walter Gordon. It is also interesting to note that in the year preceding this conference, the physicist Victor F. Weisskopf worked in the Institute of Kharkov [39] with Lev D. Landau and others, while Rudolph Peierls usually visited Landau in Leningrad 40. Both Weisskopf and Peierls knew quite well Majorana [1], the first one was met in Leipzig in 1933 (in this occasion the pair discussed on Quantum Electrodynamics), while the second one was met in Rome between the end of 1932 and the beginning of 1933, just before the visit of Majorana in Germany.

Note that even the absence of Fermi mentioned in the Majorana letter may be easily accounted by the hypothesis that the considered conference was that of Kharkov. Indeed, it is known that in the Summer of 1934 Fermi went to South America, were he held a series of conferences in Buenos Aires, Montevideo and elsewhere 33. If we let to interpret literally the words used by Majorana, the "course of conferences" seems to be more easily referred to that of 1934 in South America than to that of 1933 in the United States.

Summing up, the likely hypothesis on the date of writing of the mentioned Majorana letter in French are only two: the first one refers to the Nuclear Physics conference at Leningrad in September 1933, while the second one refers to that of Theoretical Physics at Kharkov in May 1934.

In both cases, the open problem is the possible relation between such an invitation letter to a conference in the Soviet Union and the writing of the manuscripts in the excerpta Senatore II. Unfortunately, to this regard, no conclusive supporting evidence exists. However, by analyzing carefully the life of Majorana 1], no other occasion seems to imply the possible usage of the French as possible communication medium, so that it would be likely that some a link between the two documents exists. If this conjecture is confirmed, then we can reasonably conclude that the text present in the excerpta Senatore II should be prepared for a possible Majorana talk at the conference in Leningrad or in Kharkov, although he never took part to it. And, probably, his non-participation may be well related to the uncompleted development of the theory present in the excerpta that, as mentioned above, is only sketched. 


\section{Conclusions}

From a recent recognition at the Domus Galilaeana in Pisa we have realized that in the folder of the Majorana lecture notes, given by Majorana to one of his student in Naples in the March 1938, just before his disappearance, some spare papers are present in it, not belonging to the collection of the given notes. The existence of such papers was mentioned directly by that student, Gilda Senatore, some years ago, but till now their effective presence has not been effectively reported. In the present work we have thus performed, for the first time, an accurate analysis of the cited manuscript (excerpta Senatore II), written by Majorana in French. It is the only scientific manuscript written by the author in that language. The content of it sketches a theory about Quantum Electrodynamics, with the use of the field quantization formalism. In particular, he considers some questions regarding the hole theory and the formulation of Electrodynamics suggested by Oppenheimer in analogy to the relativistic Dirac theory of the electron.

From what discussed above, it seems likely to suppose that the text considered here was elaborated in 1933-1934, probably for a conference in the Soviet Union (at Leningrad in 1933 or Kharkov in 1934) to which Majorana, though invited to participate, did not take part to it. In any case, we can reasonably exclude a date of composition far from the period when Majorana stayed in Leipzig with Heisenberg, since it seems well documented a certain dependence of the Majorana paper on an article by the German physicist.

On the other hand, as noted by Weisskopf, who knew Majorana in Leipzig, «at that time I was still interested in Quantum Electrodynamics, and there were two problems to be faced: one was the problem of the positron, whether it really is contained within the Dirac equation, the problem of the charge conjugation symmetry, as we say today, and two, the problem of the nuclear force, the beginning of Nuclear Physics... All discussions focused around nuclear structure on the one hand and Quantum Electrodynamics on the other $\gg 39$.

Then Majorana, as well as other brilliant theoreticians of that period, studied actively both fundamental questions 35] 21, and the excerpta Senatore II are a further interesting proof. Nevertheless the fact remains that the theory developed in these excerpta is only sketched and, at the moment, it is not safely to conjecture on why the great theoretical physicist has not completed his work. Certainly, it is intriguing that probably Majorana kept with him such papers in Naples after several years since their writing, and that he likely gave to his student Senatore before his mysterious disappearance. Further future researches in this directions may probably put new light on this novel and fascinating question.

\section{ACKNOWLEDGMENTS}

The constant interest and the fruitful help given to me by E. Recami and A. De Gregorio deserve my sincere gratitudr, which is here fully expressed. 


\section{Appendix A. The text by Majorana}

In the following we report the English version of the text by Majorana identified as excerpta Senatore II. We point out that, apparently, the author has probably written the original text in Italian and then translated it into French or, alternatively, he did not elaborate directly the text by following the composition rules of the French language. In few places, when strictly required by clarity, we have added additional explanatory text between brackets [...].

\section{Excerpta Senatore IIA}

Let us consider a system of $p$ electrons and put the following assumptions: 1) the interaction between the particles is sufficiently small allowing to speak about individual quantum states, so that we may consider that the quantum numbers defining the configuration of the system are good quantum numbers; 2) any electron has a number $n>p$ of inner [energetic] levels, while any other level has a much greater energy. We deduce that the states of the system as a whole may be divided into two classes. The first one is composed of those configurations for which all the electrons belong to one of the inner states. Instead the second one is formed by those configurations in which at least one electron belongs to a higher level not included in the $\underline{n}$ levels already mentioned. We will also assume that it is possible, with a sufficiently degree of approximation, to neglect the interaction between the states of the two classes. In other words we will neglect the matrix elements of the energy corresponding to the coupling of different classes, so that we may consider the motion of the $p$ particles in the $n$ inner states, as if only these states exist. Then, our aim is to translate this problem into that of the motion of $n-p$ particles in the same states, such new particles representing the holes, according to the Pauli principle.

In order to reach our purpose, it is worth to adopt the formalism used in the method known as that of the quantization of the Schrödinger equation. Let us consider the Schrödinger equation:

$$
\dot{\psi}=-\frac{2 \pi i}{h} H \psi
$$

as defining a classical field, taking into account the fact that $\psi$ does not represent a single particle but, rather, a very large number of particles, in order to neglect the granular structure of matter. Then, it is useful to highlight such a part depending on the mutual interaction of the electrons in the potential appearing in the hamiltonian. Let us then put:

$$
-\frac{h}{2 \pi i} \dot{\psi}=H \psi+V \psi
$$

being

$$
V(P)=\int G\left(P, P^{\prime}\right) \psi^{*}\left(P^{\prime}\right) \psi\left(P^{\prime}\right) \mathrm{d} \tau
$$

where $G\left(P^{\prime}, P\right)$ is the potential of the forces acting between two particles located in $P$ and $P^{\prime}$. In a natural way we will consider the energy of the field as given by 
the expression

$$
\begin{aligned}
& \int \psi^{*} H \psi \mathrm{d} \tau+\frac{1}{2} \int \psi^{*} V \psi \mathrm{d} \tau \\
& =\int \psi^{*} H \psi \mathrm{d} \tau+\frac{1}{2} \iint G\left(P, P^{\prime}\right) \psi^{*}(P) \psi^{*}\left(P^{\prime}\right) \psi(P) \psi\left(P^{\prime}\right) \mathrm{d} \tau \mathrm{d} \tau^{\prime} .
\end{aligned}
$$

Let us now expand by using a set of orthogonal functions: ${ }^{4}$

$$
\begin{gathered}
\psi=\sum a_{i} \varphi_{i}, \quad \int \varphi_{i}^{*} \varphi_{k} \mathrm{~d} \tau=\delta_{i k}, \\
H \varphi_{k}=\sum_{i} H_{i k} \varphi_{i} \\
V_{i k}=\frac{1}{2} \sum_{\ell, m} O_{i \ell, k m} a_{\ell}^{*} a_{m}
\end{gathered}
$$

we can write: ${ }^{5}$

$$
\begin{gathered}
H_{i k}=\int \varphi_{i}^{*} H \varphi_{k} \mathrm{~d} \tau \\
O_{i \ell, k m}=\iint G\left(P, P^{\prime}\right) \varphi_{i}^{*}(P) \varphi_{\ell}^{*}\left(P^{\prime}\right) \varphi_{k}(P) \varphi_{m}\left(P^{\prime}\right) \mathrm{d} \tau \mathrm{d} \tau^{\prime} .
\end{gathered}
$$

Then by substituting these into the equations of motion we write:

$$
\dot{a}_{i}=-\frac{2 \pi i}{h}\left\{\sum_{k} H_{i k} a_{k}+\sum_{\ell, k, m} O_{i \ell, k m} a_{\ell}^{*} a_{k} a_{m}\right\},
$$

and, as the expression of the energy:

$$
W=\sum_{i, k} H_{i k} a_{i}^{*} a_{k}+\frac{1}{2} \sum_{i, \ell, k, m} O_{i \ell, k m} a_{i}^{*} a_{\ell}^{*} a_{k} a_{m} .
$$

Taking into account these equations, we will now give them a quantum meaning by setting

$$
\dot{a}_{i}=-\frac{2 \pi i}{h}(a W-W a), \quad \dot{a}_{i}^{*}=-\frac{2 \pi i}{h}\left(a^{*} W-W a^{*}\right),
$$

the quantities $a$ being now matrices. In order that the equations (5) be equivalent to equations (3), we easily see that the quantities $a$ should satisfy the exchange relations:

$$
\begin{aligned}
& a_{i} a_{k}^{*}-a_{k}^{*} a_{i}=\delta_{i k}, \\
& a_{i} a_{k}-a_{k} a_{i}=0, \\
& a_{i}^{*} a_{k}^{*}-a_{k}^{*} a_{i}^{*}=0 .
\end{aligned}
$$

This means to quantize [the theory] according to the classical Heisenberg rules since, indeed, the momenta conjugate to the variables $a$ are classically the quantities $a^{*}$

\footnotetext{
${ }^{4}$ In the original manuscript, the equations appearing in the following second and third line are not written just after the first equation, but in the top corner of the sheet. In the second equation the symbol $\psi_{k}$ is used in place of $\varphi_{k}$.

${ }^{5}$ The following equation is written, in the original manuscript, as

$$
H_{i k}=\int \widetilde{\psi}_{i} H \psi_{k} \mathrm{~d} \tau
$$
}


multiplied by the factor $-h / 2 \pi i$. The Heisenberg exchange relations will lead us to consider particles obeying to the Bose statistics, while we are interested in the other case, namely that of particles obeying the Fermi statistics. As proved by Jordan and Wigner, to this end we have to change the signs in the Heisenberg relations:

$$
\begin{aligned}
& a_{i} a_{k}^{*}+a_{k}^{*} a_{i}=\delta_{i k}, \\
& a_{i} a_{k}+a_{k} a_{i}=0, \\
& a_{i}^{*} a_{k}^{*}+a_{k}^{*} a_{i}^{*}=0 .
\end{aligned}
$$

This cannot be justified on general grounds, but only by the particular form of the hamiltonian. In fact, we may verify that the equations of motion are satisfied to the best by these last exchange relations rather than by the Heisenberg ones. We now consider a suitable solution.

$$
\begin{gathered}
I_{k}=\left|\begin{array}{ll}
1 & 0 \\
0 & 1
\end{array}\right|, \quad I_{k}^{\prime}=\left|\begin{array}{cc}
1 & 0 \\
0 & -1
\end{array}\right| ; \quad \alpha_{k}=\left|\begin{array}{cc}
0 & 1 \\
0 & 0
\end{array}\right|, \quad \alpha_{k}^{*}=\left|\begin{array}{cc}
0 & 0 \\
1 & 0
\end{array}\right| \\
a_{i}=I_{1}^{\prime} \times I_{2}^{\prime} \ldots I_{i-1}^{\prime} \times \alpha_{i} \times I_{i+1} \times I_{i+2} \ldots \\
a_{i}^{*}=I_{1}^{\prime} \times I_{2}^{\prime} \ldots I_{i-1}^{\prime} \times \alpha_{i}^{*} \times I_{i+1} \times I_{i+2} \ldots \\
\alpha_{i}^{*} \alpha_{i}=\left|\begin{array}{cc}
0 & 0 \\
0 & 1
\end{array}\right|
\end{gathered}
$$

[whose eigenvalues are] 0,1 .

$$
\int \psi^{*} \psi \mathrm{d} \tau=\sum_{i} \alpha_{i}^{*} \alpha_{i}=n .
$$

6

[By setting] $b_{i}=a_{i}^{*}, b_{i}^{*}=a_{i}$, [the relation]

[can be written as]

$$
a_{i}^{*} a_{i}+a_{i} a_{i}^{*}=1
$$

$$
a_{i}^{*} a_{i}+b_{i}^{*} b_{i}=1
$$

[that is]

$$
a_{i}^{*} a_{i}=1, \quad b_{i}^{*} b_{i}=0,
$$

[or $]$

$$
a_{i}^{*} a_{i}=0, \quad \quad b_{i}^{*} b_{i}=1 .
$$

[For the energy $W$ we then have:]

[or]

$$
\sum H_{i k} a_{i}^{*} a_{k}+\frac{1}{2} \sum O_{i \ell, k m} a_{i}^{*} a_{\ell}^{*} a_{k} a_{m}
$$

\footnotetext{
${ }^{6}$ In the remaining part of the manuscript, some attempts are carried out by the author in order to calculate the matrix elements of the energy $W$ (and, in particular, of $H_{i k}$ and $O_{i \ell, k m}$ ) in the basis of the occupation numbers $n_{1}, n_{2}, \ldots, n_{i}, \ldots, n_{k}, \ldots$ Here we do not report such attempts, which are difficult to interpret.
} 


$$
\sum H_{i k} b_{i} b_{k}^{*}+\frac{1}{2} \sum O_{i \ell, k m} b_{i} b_{\ell} b_{k}^{*} b_{m}^{*}
$$

[From the relation]

$$
\begin{aligned}
& b_{i}^{*} b_{k}+b_{k}^{*} b_{i}=\delta_{i k}, \\
& b_{i}^{*} b_{k}=\delta_{i k}-b_{k}^{*} b_{i},
\end{aligned}
$$

[the first term of the energy is written as]

$$
\sum H_{i i}-\sum \bar{H}_{i k} b_{i}^{*} b_{k}
$$

[For the second term, by using:]

$$
\begin{aligned}
b_{i} b_{\ell} b_{k}^{*} b_{m}^{*} & =b_{i}\left(\delta \ell k-b_{k}^{*} b_{\ell}\right) b_{m}^{*} \\
& =\delta_{\ell k} b_{i} b_{m}^{*}-b_{i} b_{k}^{*} b_{\ell} b_{m}^{*}=\ldots \\
& =b_{k}^{*} b_{m}^{*} b_{i} b_{\ell}+\delta_{\ell k} b_{i} b_{m}^{*}+\delta_{i m} b_{\ell} b_{k}^{*}-\delta_{i k} b_{\ell} b_{m}^{*}-\delta_{\ell m} b_{i} b_{k}^{*}
\end{aligned}
$$

7

[we get:]

$$
\begin{aligned}
\frac{1}{2} \sum O_{i \ell, k m} b_{i} b_{\ell} b_{k}^{*} b_{m}^{*} & =\frac{1}{2} \sum O_{i \ell, k m} b_{k}^{*} b_{m}^{*} b_{i} b_{\ell} \\
& +\frac{1}{2} \sum O_{i \ell, \ell m} b_{i} b_{m}^{*}+\frac{1}{2} \sum O_{i \ell, k i} b_{\ell} b_{k}^{*}+\ldots
\end{aligned}
$$

8

\section{Excerpta Senatore IIb}

These $^{9}$ equations, however, satisfy a fourth one.

Then Opp. ${ }^{10}$ has attempted to build a theory with three vector components. [The Maxwell equations in vacuum:]

$$
\begin{array}{cl}
\frac{1}{c} \frac{\partial E}{\partial t}=\operatorname{rot} H, & \frac{1}{c} \frac{\partial H}{\partial t}=-\operatorname{rot} E \\
\operatorname{div} E=0, & \operatorname{div} H=0
\end{array}
$$

[by putting]

\footnotetext{
${ }^{7}$ In the last relation the author has omitted the term $\delta_{\ell m} \delta_{i k}-\delta_{i m} \delta_{\ell k}$ which, in the sum appearing in the expression of the energy, leads to a vanishing contribution.

${ }^{8}$ The text contained in the original manuscript ends with few other calculations which we do not report.

${ }^{9}$ The beginning of this part of the manuscript is evidently the continuation of a previous one, which is lost. However, see what the author writes in the following

${ }^{10}$ Probably, Majorana refers here to the paper by R.J. Oppenheimer in Phys. Rev. 38 (1931) 725 .
} 


$$
\begin{aligned}
& \psi_{x}=E_{x}-i H_{x} \\
& \psi_{y}=E_{y}-i H_{y}, \\
& \psi_{z}=E_{z}-i H_{z}
\end{aligned}
$$

[may be written in the following form:]

$$
\begin{aligned}
& \frac{1}{c} \frac{\partial \psi}{\partial t}=\operatorname{rot}(H+i E)=i \operatorname{rot}(E-i H)=i \operatorname{rot} \psi, \\
& \left\{\begin{array}{l}
\frac{1}{c} \frac{\partial \psi}{\partial t}-i \operatorname{rot} \psi=0 \\
\operatorname{div} \psi=0
\end{array}\right. \\
& \left\{\begin{array}{l}
\frac{1}{c} \frac{\partial \psi_{x}}{\partial t}-i \frac{\partial \psi_{z}}{\partial y}+i \frac{\partial \psi_{y}}{\partial z}=0 \\
\cdots \\
\frac{\partial \psi_{x}}{\partial x}+\frac{\partial \psi_{y}}{\partial y}+\frac{\partial \psi_{z}}{\partial z}=0
\end{array}\right.
\end{aligned}
$$

[By introducing the operators]

$$
W=-\frac{h}{2 \pi i} \frac{\partial}{\partial t}, \quad p_{x}=\frac{h}{2 \pi i} \frac{\partial}{\partial x}
$$

[we have:]

$$
\left\{\begin{aligned}
\frac{1}{c} W \psi_{x}+i p_{y} \psi_{z}-i p_{z} \psi_{y} & =0, \\
\frac{1}{c} W \psi_{y}+i p_{z} \psi_{x}-i p_{x} \psi_{z} & =0, \\
\frac{1}{c} W \psi_{z}+i p_{x} \psi_{y}-i p_{y} \psi_{x} & =0,
\end{aligned} \quad \underline{p_{x} \psi_{x}+p_{y} \psi_{y}+p_{z} \psi_{z}=0,}\right.
$$

[and the Maxwell equations may be written in the compact form, analogous to that of the Dirac equation,]

$$
\left[\frac{1}{c} W+(\alpha, p)\right] \psi=0
$$

[with]

$$
\alpha_{x}=\left(\begin{array}{ccc}
0 & 0 & 0 \\
0 & 0 & -i \\
0 & i & 0
\end{array}\right), \quad \alpha_{y}=\left(\begin{array}{ccc}
0 & 0 & i \\
0 & 0 & 0 \\
-i & 0 & 0
\end{array}\right), \quad \alpha_{z}=\left(\begin{array}{ccc}
0 & -i & 0 \\
i & 0 & 0 \\
0 & 0 & 0
\end{array}\right)
$$


[In order that the equation satisfied by $\psi$ have a non trivial solution, it needs that the energy $W$ is given by]

$$
\frac{W}{c}=\left(\begin{array}{ccc}
0 & i p_{z} & -i p_{y} \\
-i p_{z} & 0 & i p_{x} \\
i p_{y} & -i p_{x} & 0
\end{array}\right)
$$

[whose eigenvalues are]

$$
\frac{W}{c}=p, \quad \frac{W}{c}=-p, \quad \frac{W}{c}=0
$$

[while the ratio between the components of the eigenvector corresponding to the null eigenvalue is given by:]

$$
\begin{gathered}
\psi_{x}: \psi_{y}: \psi_{z}=-p_{x}^{2}:-p_{x} p_{y}:-p_{x} p_{z}=p_{x}: p_{y}: p_{z} \\
\widetilde{\psi} \psi=E^{2}+B^{2} .
\end{gathered}
$$

\section{Excerpta Senatore IIC}

Oppenheimer has tried to build such equations with an inductive procedure by using the experimental properties of the photons.

These properties may be summarized in the following way:

1) the photons move at the speed of light;

2 ) the energy and the momentum are related each other by the very simple relation: $W=c p$;

3) given the strength and the direction of the momentum, two possible polarization states are present;

4) the photon has a spin that may assume the values $\pm \frac{h}{2 \pi}$ along the propagation axis. For an experimental justification of this last postulate, we observe that, when an atom emits some radiation, its angular momentum around a given $z$ axis changes from + to $-\frac{h}{2 \pi}$ (or 0 ) depending on the fact that one or the other of the following transitions takes place:

$$
\begin{gathered}
m \rightarrow m^{\prime}=m \mp 1 \\
\left(m \rightarrow m^{\prime}=m\right) .
\end{gathered}
$$

Then, if we observe a photon along the $z$ axis, we can certainly say that such a photon cannot be due to the last transition, since the intensity of this vanishes along the $[z]$ axis. On the other hand if the photon lies exactly on the $z$ axis, this cannot depend on the angular momentum around this axis, so that from the conservation of the angular momentum of the system we must admit the existence of the spin of the photon, in a way that it may assume the two values $\pm \frac{h}{2 \pi}$ along the propagation direction. Such an interpretation of the spin of the photon has been proposed for 
the first time by Dirac, when he was not yet R.R.S. ${ }^{11}$

Since we are concerned with two polarized components, it would be worth to consider a two-component wave theory. Indeed we can build some equations of the desired form satisfying to the first three postulates. These equations are the following ones:

$$
\left[\frac{W}{c}+(\sigma, p)\right] \psi=0 ; \quad \sigma_{x}=\left|\begin{array}{cc}
0 & 1 \\
1 & 0
\end{array}\right|, \quad \sigma_{y}=\left|\begin{array}{cc}
0 & -i \\
i & 0
\end{array}\right|, \quad \sigma_{z}=\left|\begin{array}{cc}
1 & 0 \\
0 & -1
\end{array}\right| .
$$

I point out that these equations are nothing other that half of the Dirac equation.

\section{REFERENCES}

[1] E. Recami, Il caso Majorana (Oscar Mondadori, Milan, 1991, second edition); idem (Di Renzo, Rome, 2002, fourth revised and enlarged edition).

[2] S. Esposito, Physics World 19 (2006) 34,

[3] A. De Gregorio and S. Esposito, Sapere, Giugno 2006, p. 56 arXiv:physics/0602008; A. De Gregorio and S. Esposito, arXiv:physics/0602146.

[4] S. Esposito, Nuovo Saggiatore 21 (2005) 21; A. Drago and S. Esposito, Phys. Persp., in press arXiv:physics/0503084.

[5] A. Drago and S. Esposito, Found. Phys. 34 (2004) 871.

[6] G. Senatore, Lecture, Department of Physical Sciences, University of Naples "Federico II", March 30, 1998.

[7] E. Majorana, Lezioni all'Università di Napoli, (Bibliopolis, Naples, 1987).

[8] E. Majorana, Lezioni di Fisica Teorica, edited by S. Esposito (Bibliopolis, Naples, 2006).

[9] S. Esposito, Eur. J. Phys. 27 (2006) 1147 arXiv:physics/0603140; S. Esposito, arXiv:physics/0512174.

[10] W. Heisenberg, Naturwissenschaften 26 (1929) 490.

[11] P. Jordan, Anscauliche Quantentheorie eine Einführung in die moderne Auffassung der Quantenenerscheinurgen (Springer, Berlin, 1926).

[12] P.A.M. Dirac, Proc. Roy. Soc. London 114 (1927) 243, 710.

[13] P. Jordan and O. Klein, Z. Phys. 45 (1927) 755.

[14] P. Jordan and E. Wigner, Z. Phys. 47 (1928) 631.

[15] W. Heisenberg and W. Pauli, Z. Phys. 56 (1929) 1, ibidem 59 (1930) 168.

[16] I. Waller, Z. Phys. 61 (1930) 837.

[17] P.A.M. Dirac, Proc. Roy. Soc. London 126 (1930) 360; Proc. Cambridge. Phil. Soc. 26 (1930) 361.

[18] H. Weyl, Z. Phys. 56 (1929) 330.

[19] W. Pauli, Handbuch der Physik, 24 (1933) 83. See also the letters by W. Pauli to P.M. Blackett (19 April 1933) and to P.A.M. Dirac (1 May 1933) reported in W. Pauli, Wissenschaften Briefwechsel mit Bohr, Einstein, Heisenberg, U.A. II: 1930-1939 (Springer, Berlin, 1985).

[20] See the letter by W. Heisenberg to A. Sommerfeld (17 June 1933) reported in W. Pauli, Wissenschaften ..., loc. cit.

[21] E. Majorana, Nuovo Cim. 14 (1937) 171.

[22] W. Heisenberg, Ann. der Physik 10 (1931) 888.

[23] See the list of the scientific unpublished manuscript reported in E. Recami, Quaderni di Storia della Fisica, 5 (1999) 19.

[24] J.R. Oppenheimer, Phys. Rev. 38 (1931) 725.

[25] R. Mignani, E. Recami and M. Baldo, Lett. Nuovo Cim. 11 (1974) 568; E. Giannetto, Lett. Nuovo Cim. 44 (1985) 140 and 145; E. Giannetto, in F. Bevilacqua (ed.) Atti IX Congresso Naz.le di Storia della Fisica (Milan, 1988) p.173. S. Esposito, Found. Phys. 28 (1998) 231.

[26] E. Majorana, Nuovo Cim. 9 (1932) 43.

[27] G. Gentile and E. Majorana, Rend. Acc. Lincei 8 (1928) 229.

\footnotetext{
${ }^{11}$ Probably such initials should be corrected in F.R.S., Fellow of the Royal Society. In this way, the author points out that the work by Dirac was performed before his election as a member of the Royal Society of London (in March 1930). In any case, it is quite interesting to find such an historical note in a scientific manuscript by Majorana.
} 
[28] E. Fermi and B. Rossi, Rend. Acc. Lincei 17 (1933) 346.

[29] E. Fermi, Collected Papers, edited by E. Amaldi et al., University of Chicago Press - Accademia Nazionale dei Lincei, Chicago - Rome, 1962-1965, 2 volumes, Vol. I (1962).

[30] M.P. Bronshtein, V.M. Dukel'skii, D.D. Ivanenko and Yu. B. Khariton (eds.), Problems of Modern Physics Vol. 24: The Atomic Nucleus: Collected Papers of the First All Union Nuclear Conference, held 24-30 September 1933 at Leningrad, Leningrad and Moscow State Technical-Theoretical Publishing House, 1934.

[31] V.P. Vizgin, Physics Uspekhi 42 (1999) 1259.

[32] P.A.M. Dirac, in M.P. Bronshtein, V.M. Dukel'skii, D.D. Ivanenko and Yu. B. Khariton (eds.), loc. cit., p. 129.

[33] E. Segré, Enrico Fermi Physicist (The University of Chicago Press, Chicago, 1970).

[34] See the date reported in E. Fermi and G.E. Uhlenbeck, Phys. Rev. 44 (1933) 510.

[35] E. Majorana, Z. Phys. 82 (1933) 137; Ricerca Scientifica 4 (1933) 559.

[36] A. De Gregorio, Stud. Hist. Philos. Mod. Phys. 37 (2006) 330; A. De Gregorio, arXiv:physics/0603261.

[37] E. L. Feinberg, Physics Uspekhi 38 (1995) 773.

[38] G. Waysand, Phys. Stat. Sol. C2 (2005) 1566.

[39] V.F. Weisskopf, talk given at the Erice Summer School in High-Energy Physics, Erice (Italy), 1971.

[40] See the Chronology of the Life of Sir Rudolph Ernst Peierls in R.H. Dalitz and R. Peierls, Selected Scientific Papers of Sir Rudolf Peierls with commentary (World Scientific - Imperial College Press, Singapore - London, 1997).

S. Esposito: Dipartimento di Scienze Fisiche, Università di Napoli "Federico II" \& I.N.F.N. Sezione di Napoli, Complesso Universitario di M. S. Angelo, Via Cinthia, 80126 NAPOLI (Salvatore.Esposito@na.infn.it) 